\title{
Crystal structure of 3,6-bis(4-biphenylyl)pyrrolo[3,4-c]pyrrole-1,4-dione, $\mathrm{C}_{30} \mathrm{H}_{20} \mathrm{~N}_{2} \mathrm{O}_{2}$
}

\author{
J. Mizuguchi ${ }^{* . I}$ and T. Miyazaki ${ }^{I I}$ \\ 1 Yokohama National University. Graduate School of Engineering. Department of Applied Physics. 79-5 Tokiwadai. Hodogaya-ku. 240-8501 Yokohama, Japan \\ II Yokohama National University. Faculty of Education. 79.2 Tokiwadai. Hodogaya-ku. 240.8501 Yokohama. Japan
}

Received September 9. 2001. accepted December 12. 2001: CCDC-No. 1267/725

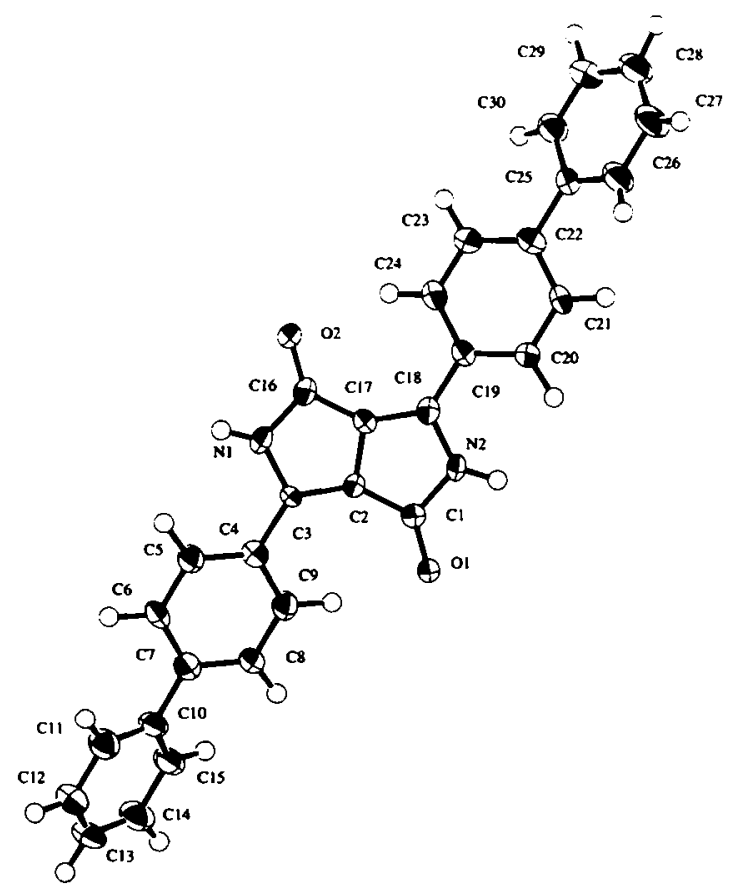

Abstract

$\mathrm{C}_{30} \mathrm{H}_{20} \mathrm{~N}_{2} \mathrm{O}_{2}$, triclinic, $P \overline{1}$ (No. 2), $a=7.226(2) \AA, b=21.886(4) \AA$, $c=6.793(5) \AA, \alpha=95.25(3)^{\circ}, \beta=90.18(4)^{\circ}, \gamma=93.82(2)^{\circ}$,

$V=1067.4 \AA^{3}, Z=2, R_{\mathrm{gt}}(F)=0.073, w R_{\text {ref }}\left(F^{2}\right)=0.201, T=253 \mathrm{~K}$.

\section{Source of material}

The title compound was obtained from CIBA Specialty Chemicals. The sample was purified by sublimation at about $620 \mathrm{~K}$, using a two-zone furnace [1]. After $60 \mathrm{~h}$, a number of red platelet crystals were obtained.

\section{Experimental details}

The quality of the crystal studied was not sufficiently high, so that only $45 \%$ of the measured reflections could be considered as observed $\left(I_{\mathrm{obs}}>3 \sigma\left(I_{\mathrm{obs}}\right)\right)$. This is also reflected in the final relative high $R$-values.

\section{Discussion}

Diketopyrrolopyrrole (abbreviated to DPP) pigments are industrially important red pigments based on the novel diketopyrrolopyrrole chromophore [2]. DPPs are also used as colorants for imaging areas as well as a color filter for LCD applications. Up to now, five DPP-derivatives are on the market and most of their

\footnotetext{
* Correspondence author (e-mail: mizu-j@ynu.ac.jp)
}

structures have already been analyzed [3-6]. The title compound (BP-DPP) is characterized by an extremely large bathochromic shift upon crystallization as compared with other DPP analogues. The present structure analysis has therefore been carried out in order to clarify the electronic structure of BP-DPP. As shown in the figure, the molecule is deformed as characterized by molecular symmetry of $C_{1}$. The two phenyl rings on each side of the heterocyclic ring system are twisted asymmetrically in opposite directions. In addition, the heterocyclic ring system is not entirely planar, but is folded in the middle with a dihedral angle of $175.7^{\circ}$. Due to this deformed structure, a dipole moment of about $0.18 \mathrm{D}$ appears perpendicular to the molecular plane. The molecules are arranged in a fashion "bricks in a brick wall", just like J-aggregates in cyanine dyestuffs [7]. There are two kinds of stacking columns: one is composed of the molecules with an upward dipole moment while the neighboring column comprises the molecules with a downward dipole moment. The polar molecules are stacked pairwise so as to reduce the electrostatic energy. On the other hand, on the molecular plane along the $a$ axis, there are chains of intermolecular hydrogen bonds between the $\mathrm{NH}$ group of one molecule and the $\mathrm{O}$ atom of the neighboring one. This forms a two-dimensional hydrogen bond network as found in all DPP pigments [3-6]. Details of the correlation between the crystal and electronic structures are given in references $[8,9]$.

Table 1. Data collection and handling.

\section{Crystal:}

Wavelength:

$\mu$ :

Diffractometer, scan mode:

$2 \theta_{\max }$ :

$N(h k l)_{\text {measured, }} N(h k l)_{\text {unique: }}$

Criterion for $I_{\mathrm{obs}}, N(h k l)_{\mathrm{gg}}$ :

$N($ param) refined:

Programs:

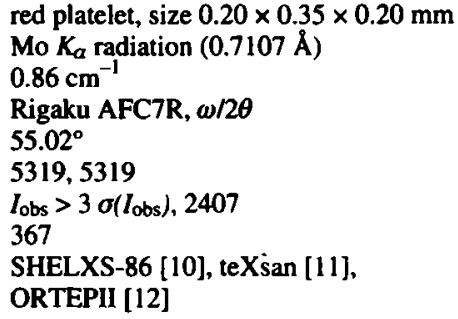

Table 2. Atomic coordinates and displacement parameters (in $\AA^{2}$ ).

\begin{tabular}{lllrrl}
\hline Atom & Site & \multicolumn{1}{l}{$\boldsymbol{x}$} & \multicolumn{1}{l}{$\boldsymbol{y}$} & \multicolumn{1}{l}{$U_{\text {iso }}$} \\
\hline $\mathbf{H}(1)$ & $2 i$ & $0.34(1)$ & $-0.042(3)$ & $0.21(1)$ & 0.045 \\
$\mathbf{H}(2)$ & $2 i$ & $1.15(1)$ & $0.044(3)$ & $0.29(1)$ & 0.045 \\
$\mathbf{H}(3)$ & $2 i$ & $0.31(1)$ & $-0.149(3)$ & $0.17(1)$ & 0.045 \\
$\mathbf{H}(4)$ & $2 i$ & $0.281(9)$ & $-0.253(3)$ & $0.04(1)$ & 0.045 \\
$\mathbf{H}(5)$ & $2 i$ & $0.84(1)$ & $-0.239(3)$ & $-0.10(1)$ & 0.045 \\
$\mathbf{H}(6)$ & $2 i$ & $0.870(9)$ & $-0.135(3)$ & $0.01(1)$ & 0.045 \\
$\mathbf{H}(7)$ & $2 i$ & $0.338(9)$ & $-0.354(3)$ & $0.09(1)$ & 0.045 \\
$\mathbf{H}(8)$ & $2 i$ & $0.321(9)$ & $-0.458(3)$ & $-0.01(1)$ & 0.045 \\
\hline
\end{tabular}


Table 2. Continued.

\begin{tabular}{llllll}
\hline Alom & Site & $x$ & $y$ & $z$ & $U_{\text {iso }}$ \\
\hline H(9) & $2 i$ & $0.50(1)$ & $-0.492(4)$ & $-0.30(1)$ & 0.045 \\
H(10) & $2 i$ & $0.73(1)$ & $-0.418(3)$ & $-0.44(1)$ & 0.045 \\
H(11) & $2 i$ & $0.732(9)$ & $-0.319(3)$ & $-0.33(1)$ & 0.045 \\
H(12) & $2 i$ & $1.19(1)$ & $0.136(3)$ & $0.50(1)$ & 0.045 \\
H(13) & $2 i$ & $1.196(9)$ & $0.244(3)$ & $0.61(1)$ & 0.045 \\
H(14) & $2 i$ & $0.654(9)$ & $0.259(3)$ & $0.44(1)$ & 0.045 \\
\hline
\end{tabular}

Table 2. Continued.

\begin{tabular}{llllll}
\hline Atom & Site & $x$ & $y$ & $z$ & $U_{\text {iso }}$ \\
\hline $\mathrm{H}(15)$ & $2 i$ & $\mathbf{0 . 6 2 5 ( 9 )}$ & $0.154(3)$ & $\mathbf{0 . 3 3 ( 1 )}$ & 0.045 \\
$\mathrm{H}(16)$ & $2 i$ & $1.117(9)$ & $0.316(3)$ & $0.85(1)$ & 0.045 \\
$\mathrm{H}(17)$ & $2 i$ & $1.15(1)$ & $0.416(3)$ & $0.97(1)$ & 0.050 \\
$\mathrm{H}(18)$ & $2 i$ & $\mathbf{0 . 9 9 ( 1 )}$ & $0.499(4)$ & $\mathbf{0 . 7 7 ( 1 )}$ & 0.048 \\
$\mathrm{H}(19)$ & $2 i$ & $\mathbf{0 . 8 0 6 ( 9 )}$ & $0.464(3)$ & $\mathbf{0 . 5 2 ( 1 )}$ & 0.046 \\
$\mathrm{H}(20)$ & $2 i$ & $0.789(9)$ & $0.354(3)$ & $\mathbf{0 . 3 9 ( 1 )}$ & 0.045 \\
\hline
\end{tabular}

Table 3. Atomic coordinates and displacement parameters (in $\AA^{2}$ ).

\begin{tabular}{|c|c|c|c|c|c|c|c|c|c|c|}
\hline Atom & Site & $x$ & $y$ & $z$ & $U_{11}$ & $U_{22}$ & $U_{33}$ & $U_{12}$ & $U_{13}$ & $U_{23}$ \\
\hline$O(1)$ & $2 i$ & $1.0836(5)$ & $-0.0653(2)$ & $0.1742(7)$ & $0.018(2)$ & $0.019(2)$ & $0.043(3)$ & $0.001(2)$ & $0.000(2)$ & $-0.004(2)$ \\
\hline$O(2)$ & $2 i$ & $0.4046(5)$ & $0.0683(2)$ & $0.3275(7)$ & $0.017(2)$ & $0.023(2)$ & $0.047(3)$ & $0.002(2)$ & $0.001(2)$ & $-0.005(2)$ \\
\hline$N(1)$ & $2 i$ & $0.4638(7)$ & $-0.0323(3)$ & $0.2194(8)$ & $0.014(3)$ & $0.028(3)$ & $0.037(3)$ & $0.002(2)$ & $0.001(2)$ & $-0.002(2)$ \\
\hline $\mathbf{N}(2)$ & $2 i$ & $1.0245(7)$ & $0.0348(2)$ & $0.2910(8)$ & $0.013(2)$ & $0.024(3)$ & $0.030(3)$ & $-0.002(2)$ & $0.000(2)$ & $-0.001(2)$ \\
\hline$C(1)$ & $2 i$ & $0.9714(8)$ & $-0.0255(3)$ & $0.2151(9)$ & $0.020(3)$ & $0.020(3)$ & $0.031(4)$ & $0.002(2)$ & $0.001(2)$ & $0.001(3)$ \\
\hline $\mathrm{C}(2)$ & $2 i$ & $0.7718(8)$ & $-0.0273(3)$ & $0.2104(9)$ & $0.016(3)$ & $0.020(3)$ & $0.029(3)$ & $0.001(2)$ & $-0.001(2)$ & $0.003(3)$ \\
\hline$C(3)$ & $2 i$ & $0.6145(8)$ & $-0.0665(3)$ & $0.1747(9)$ & $0.018(3)$ & $0.013(3)$ & $0.029(4)$ & $0.000(2)$ & $-0.003(2)$ & $-0.001(2)$ \\
\hline$C(4)$ & $2 i$ & $0.5925(9)$ & $-0.1318(3)$ & $0.1046(9)$ & $0.026(3)$ & $0.022(3)$ & $0.019(3)$ & $0.000(3)$ & $0.002(2)$ & $0.002(2)$ \\
\hline$C(5)$ & $2 i$ & $0.4238(9)$ & $-0.1662(3)$ & $0.120(1)$ & $0.023(3)$ & $0.025(3)$ & $0.039(4)$ & $-0.001(2)$ & $0.007(3)$ & $-0.004(3)$ \\
\hline$C(6)$ & $2 i$ & $0.4082(9)$ & $-0.2285(3)$ & $0.050(1)$ & $0.027(3)$ & $0.018(3)$ & $0.038(4)$ & $-0.005(2)$ & $0.001(3)$ & $-0.002(3)$ \\
\hline $\mathrm{C}(7)$ & $2 i$ & $0.5577(9)$ & $-0.2578(3)$ & $-0.0351(9)$ & $0.027(3)$ & $0.023(3)$ & $0.025(3)$ & $-0.001(3)$ & $-0.002(3)$ & $0.003(3)$ \\
\hline$C(8)$ & $2 i$ & $0.7272(8)$ & $-0.2227(3)$ & $-0.049(1)$ & $0.024(3)$ & $0.020(3)$ & $0.034(4)$ & $0.000(2)$ & $0.001(3)$ & $-0.003(3)$ \\
\hline C(9) & $2 i$ & $0.7446(8)$ & $-0.1605(3)$ & $0.022(1)$ & $0.022(3)$ & $0.028(3)$ & $0.032(4)$ & $-0.001(2)$ & $0.002(3)$ & $0.000(3)$ \\
\hline$C(10)$ & $2 i$ & $0.5419(9)$ & $-0.3236(3)$ & $-0.1091(9)$ & $0.032(4)$ & $0.019(3)$ & $0.031(4)$ & $0.001(3)$ & $0.000(3)$ & $-0.001(3)$ \\
\hline$C(11)$ & $2 i$ & $0.4255(9)$ & $-0.3661(3)$ & $-0.018(1)$ & $0.035(3)$ & $0.029(3)$ & $0.035(4)$ & $0.000(3)$ & $0.005(3)$ & $-0.002(3)$ \\
\hline$C(12)$ & $2 i$ & $0.412(1)$ & $-0.4276(3)$ & $-0.086(1)$ & $0.042(4)$ & $0.024(3)$ & $0.052(5)$ & $-0.003(3)$ & $-0.003(3)$ & $0.002(3)$ \\
\hline$C(13)$ & $2 i$ & $0.514(1)$ & $-0.4487(3)$ & $-0.248(1)$ & $0.047(4)$ & $0.019(3)$ & $0.048(4)$ & $0.001(3)$ & $-0.003(3)$ & $-0.006(3)$ \\
\hline$C(14)$ & $2 i$ & $0.628(1)$ & $-0.4074(3)$ & $-0.341(1)$ & $0.046(4)$ & $0.028(4)$ & $0.041(4)$ & $0.003(3)$ & $0.003(3)$ & $-0.007(3)$ \\
\hline$C(15)$ & $2 i$ & $0.6421(9)$ & $-0.3452(3)$ & $-0.272(1)$ & $0.037(4)$ & $0.021(3)$ & $0.046(4)$ & $-0.001(3)$ & $0.008(3)$ & $-0.001(3)$ \\
\hline$C(16)$ & $2 i$ & $0.5181(8)$ & $0.0289(3)$ & $0.2817(9)$ & $0.017(3)$ & $0.027(3)$ & $0.022(3)$ & $-0.001(2)$ & $0.001(2)$ & $0.002(2)$ \\
\hline$C(17)$ & $2 i$ & $0.7174(8)$ & $0.0318(3)$ & $0.2764(9)$ & $0.021(3)$ & $0.018(3)$ & $0.029(4)$ & $-0.001(2)$ & $0.000(2)$ & $-0.001(3)$ \\
\hline$C(18)$ & $2 i$ & $0.8756(8)$ & $0.0700(3)$ & $0.3247(9)$ & $0.019(3)$ & $0.025(3)$ & $0.022(3)$ & $-0.001(2)$ & $0.000(2)$ & $0.001(3)$ \\
\hline$C(19)$ & $2 i$ & $0.8954(9)$ & $0.1345(3)$ & $0.398(1)$ & $0.023(3)$ & $0.019(3)$ & $0.030(4)$ & $-0.002(2)$ & $-0.001(3)$ & $-0.003(3)$ \\
\hline$C(20)$ & $2 i$ & $1.0644(8)$ & $0.1609(3)$ & $0.4793(9)$ & $0.022(3)$ & $0.020(3)$ & $0.033(4)$ & $0.002(2)$ & $-0.003(3)$ & $0.001(3)$ \\
\hline $\mathrm{C}(21)$ & $2 i$ & $1.0830(8)$ & $0.2228(3)$ & $0.549(1)$ & $0.025(3)$ & $0.022(3)$ & $0.034(4)$ & $-0.006(2)$ & $-0.006(3)$ & $-0.003(3)$ \\
\hline $\mathrm{C}(22)$ & $2 i$ & $0.9339(9)$ & $0.2606(3)$ & $0.5358(9)$ & $0.033(4)$ & $0.020(3)$ & $0.025(3)$ & $0.001(3)$ & $0.000(3)$ & $0.000(3)$ \\
\hline $\mathrm{C}(23)$ & $2 i$ & $0.7672(9)$ & $0.2335(3)$ & $0.456(1)$ & $0.029(3)$ & $0.023(3)$ & $0.041(4)$ & $0.003(3)$ & $-0.003(3)$ & $-0.006(3)$ \\
\hline$C(24)$ & $2 i$ & $0.7471(9)$ & $0.1718(3)$ & $0.388(1)$ & $0.026(3)$ & $0.025(3)$ & $0.034(4)$ & $-0.003(2)$ & $-0.005(3)$ & $-0.001(3)$ \\
\hline $\mathrm{C}(25)$ & $2 i$ & $0.9547(8)$ & $0.3267(3)$ & $0.6118(9)$ & $0.023(3)$ & $0.022(3)$ & $0.031(4)$ & $-0.002(3)$ & $-0.002(3)$ & $-0.001(3)$ \\
\hline$C(26)$ & $2 i$ & $1.070(1)$ & $0.3467(3)$ & $0.772(1)$ & $0.042(4)$ & $0.025(3)$ & $0.042(4)$ & $-0.002(3)$ & $-0.008(3)$ & $-0.003(3)$ \\
\hline $\mathrm{C}(27)$ & $2 i$ & $1.089(1)$ & $0.4089(3)$ & $0.842(1)$ & $0.055(5)$ & $0.029(4)$ & $0.041(4)$ & $-0.001(3)$ & $-0.014(3)$ & $-0.012(3)$ \\
\hline$C(28)$ & $2 i$ & $0.992(1)$ & $0.4516(3)$ & $0.752(1)$ & $0.049(5)$ & $0.025(4)$ & $0.046(4)$ & $0.001(3)$ & $-0.005(3)$ & $-0.004(3)$ \\
\hline $\mathrm{C}(29)$ & $2 i$ & $0.879(1)$ & $0.4312(3)$ & $0.590(1)$ & $0.044(4)$ & $0.023(3)$ & $0.049(4)$ & $0.002(3)$ & $-0.001(3)$ & $-0.002(3)$ \\
\hline$C(30)$ & $2 i$ & $0.8588(9)$ & $0.3705(3)$ & $0.521(1)$ & $0.037(4)$ & $0.027(3)$ & $0.035(4)$ & $0.001(3)$ & $-0.008(3)$ & $-0.001(3)$ \\
\hline
\end{tabular}

Acknowledgments. The authors would like to express their sincere thanks to $\mathbf{N}$. Ishizuka and $\mathbf{N}$. Enomoto for experimental assistance.

\section{References}

1. Mizuguchi, J.: An improved method for purification of $\beta$-copperphtahlocya-anine. Cryst. Res. Tech. 16 (1981) 65-700.

2. Iqbal, A.; Cassar, L; Rochat, A. C.; Pfenninger, J.; Wallquist, O.: New heterocyclic pigments. J. Coat. Tech. 60 (1988) 37-45.

3. Mizuguchi, J.; Grubenmann, A.; Rihs, R.: Structures of 3,6-bis-(3chlorphenyl)pyrrolo[3,4-c]pyrrole-1,4-dione and 3,6-bis-(4-chlorophenyl)-pyrrolo[3,4-c]pyrrole-1,4-dione. Acta Crystallogr. B49 (1993) 1056-1060.

4. Mizuguchi, J.; Grubenmann, A.; Wooden, G.; Rihs, G.: Structure of 3,6diphenylpyrrolo[3,4-c]pyrrolo-1,4-dione and 3,6-dipheny1-2,5dimethylpyrrolo[3,4-c]pyrrole-1,4-dione. Acta Crystallogr. B48 (1992) 696-700.

5. Mizuguchi, J.: Structure of 3,6-bis(4-tert-butylphenyl)pyrrolo[3,4-c]pyrrole-1,4-dione. Acta Crystallogr. C54 (1998) 1482-1484.
6. Mizuguchi, J.; Matsumoto, S.: Structure of 3,6-bis(3-cyanophenyl)pyrrolo[3,4-c]pyrrole-1,4-dione. Z. Kristallogr. NCS 215 (2000) 195-196.

7. Kobayashi, T.: J-Aggregates, World Scientific Publishing Co. Pte. Ltd., 1996.

8. Mizuguchi, J.: Correlation between crystal and electronic structures in diketopyrrolopyrrole pigments as viewed from exciton coupling effects. J. Phys. Chem. A 104 (2000) 1817-1821.

9. Mizuguchi, J.: J-aggregate-like structure and its influence on the electronic spectra in 1,4-diketo-3,6-bis(4-biphenylyl)pyrrolo[3,4-c]pyrrole. J. Imag. Sci. Tech. (2001) in press.

10. Sheldrick, G. M.: Phase Annealing in SHELX-90: Direct methods for Larger Structure. Acta Crystallogr. A46 (1990) 467-473.

11. teXsan. Single Crystal Structure Analysis Software. Version 1.11. Molecular Structure Corporation, 3200 Research Forest Drive. The Woodlands, TX 77381, USA 2000.

12. Johnson, C. K.: ORTEPII, Report ORNL-5138. Oak Rodge National Laboratory, Tennessee, USA 1976. 